\title{
PROPERTIES ON THE UNIT CIRCLE OF POLYNOMIALS WITH UNIMODULAR COEFFICIENTS
}

\author{
DONALD J. NEWMAN AND ANDRÉ GIROUX
}

(Communicated by Clifford J. Earle, Jr.)

\begin{abstract}
A concrete explicit construction of a unimodular polynomial with prescribed zeros on the unit circle is given. More precisely a polynomial $P(z)=$ $a_{0}+a_{1} z+\cdots a_{N} z^{N}$ is produced for which $\left|a_{i}\right|=1$ for all $i=0,1, \ldots, N$ and for which $P\left(\alpha_{j}\right)=0$ for a given set of $\alpha_{j}, j=1,2, \ldots, n,\left|\alpha_{j}\right|=1$, and $P(z) \neq 0$ elsewhere on $|z|=1$. It is further shown how to extend this construction so as to maintain these properties and force the maximum of $|P(z)|$ to occur at any given number $\beta \neq \alpha_{j}, j=1,2, \ldots, n$ and $|\beta|=1$. The dependence of $N$ on $n$ is exponential, but there is reason to believe that this is actually necessary and not just a weakness of the method.
\end{abstract}

The following problem is posed in [1]: given the magnitude of the coefficients of a polynomial $P$, a finite subset $S$ of the unit circle $C$, and a point $p$ on $C$ distinct from those in $S$, choose the phases of the coefficients so that $P(z)=0$ for all $z$ in $S$, the maximum on $C$ of $|P(z)|$ occurs at $z=p$, and the maximum of $|P(z)|$ on a subset of $C$ excluding an appropriate interval around $p$ (the "beamwidth") is as small as possible. As explained in [1], this problem arises naturally in linear antenna theory, filter theory, and other classical electrical engineering applications.

Our main result is encompassed in the following theorem:

Theorem. Given $\alpha_{1}, \alpha_{2}, \ldots, \alpha_{n}, \beta$ on $C, \beta \neq \alpha_{i}$, there exists a polynomial $P$ with coefficients of modulus one such that the zeros of $P$ on $C$ are precisely the points $\alpha_{1}, \alpha_{2}, \ldots, \alpha_{n}$ and that

$$
\|P\| \equiv \max \{|P(z)|: z \in C\}=|P(\beta)| .
$$

(The points $\alpha_{j}$ are not assumed to be distinct: multiple zeros are allowed.)

We shall prove the theorem in two steps, first constructing a polynomial with coefficients of modulus one with the prescribed zeros (Lemma 1) and then modifying it so that it also has the prescribed maximum (Lemma 4).

Received by the editors March 6, 1989.

1980 Mathematics Subject Classification. Primary 42A16, 42 A28.

The authors are also with Temple University and the University of Montreal, respectively.

Research sponsored by the Air Force Office of Scientific Research (AFSC), under Contract F49620-88-C-0028. The United States Government is authorized to reproduce and distribute reprints for governmental purposes notwithstanding any copyright notation hereon. 
Let $U$ denote the (non-linear) class of polynomials with coefficients of modulus one. We introduce in $U$ the operation of "encapsulation" which we denote by $\otimes$.

Definition. Let $M$ be the degree of $P$. Then

$$
(P \otimes Q)(z) \equiv P(z) Q\left(z^{M+1}\right) .
$$

Explicitly, then, if $P(z)=\sum_{k=0}^{M} a_{k} z^{k}$ and $Q(z)=\sum_{j=0}^{N} b_{j} z^{j}$, then $P \otimes Q(z)=$ $\sum_{i=0}^{N(M+1)+M} c_{i} z^{i}$, where $c_{j(M+1)+k}=a_{k} b_{j}$ for $0 \leq k \leq M$ and $0 \leq j \leq N$.

From this expression, it is clear that $\otimes$ is associative and that $U$ is indeed closed under it. Also, if $Q$ does not vanish on $C$, then the zeros of $P \otimes Q$ are precisely those of $P$.

Lemma 1. Given $\alpha_{1}, \alpha_{2}, \ldots, \alpha_{n}$ on $C$, there exists $P_{n}$ in $U$ which vanishes at the $\alpha_{j}$ and nowhere else on $C$.

Proof. Let $\omega=\exp 2 \pi i / 3$. We shall prove a little more, namely that such a polynomial can be found with constant term one and leading term $-\omega$. We use induction on $n$. Let

$$
Q_{1}(z)=1-z / \alpha_{1}
$$

and let

$$
P_{1}(z)=Q_{1}(z) \quad \text { if } \alpha_{1} \omega=1
$$

and

$$
\begin{aligned}
P_{1}(z) & =Q_{1}(z) \otimes\left(1+z+\alpha_{1} \omega z^{2}\right) \\
& =1-\left(1 / \alpha_{1}\right) z+z^{2}-\left(1 / \alpha_{1}\right) z^{3}+\alpha_{1} \omega z^{4}-\omega z^{5}
\end{aligned}
$$

otherwise (the condition $\alpha_{1} \omega \neq 1$ also ensures that the polynomial $1+z+$ $\alpha_{1} \omega z^{2} \neq 0$ on $C$ ). Assume now that $R(z)=1+\cdots-\omega z^{N}$ is a polynomial in $U$ of which the zeros on $C$ are precisely the points $\beta_{j}=\alpha_{j} / \alpha_{n}$ for $1 \leq j \leq n-1$. Set

$$
\begin{aligned}
S(z) & =R(z)\left(1-z^{N}+z^{2 N}-z^{3 N+1}\right) \\
& =1+\cdots+\omega z^{4 N+1} .
\end{aligned}
$$

To verify that $S$ is in $U$, we need only check the modulus of the coefficients of $z^{N}$ and $z^{2 N}$. But, the coefficient of $z^{N}$ is $-\omega-1=\omega^{2}$ and that of $z^{2 N}$ is $\omega+1=-\omega^{2}$. We now claim that

$$
1-z^{N}+z^{2 N}-z^{3 N+1}
$$

does not vanish on $C$ except for a simple zero at $z=1$. Indeed, for the sum of four points on $C$ to vanish, it is necessary and sufficient that they cancel in pairs. There are thus three possibilities:

(a) $1-z^{N}=0$ and $z^{2 N}-z^{3 N+1}=0$; this gives $z^{N}=1$ and $z^{N+1}=1$, hence $z=1$; 
(b) $1+z^{2 N}=0,-z^{N}-z^{3 N+1}=0$; this implies $z^{2 N}=-1, z^{2 N+1}=-1$, which is impossible;

(c) $1-z^{3 N+1}=0,-z^{N}+z^{2 N}=0$; in this case, $z^{3 N+1}=1$ and $z^{N}=1$; therefore $z^{2 N+1}=z^{N+1}=z^{N}=1$, hence $z=1$ again.

Setting

$$
Q_{n}(z)=S\left(z / \alpha_{n}\right)=1+\cdots+\left(\omega / \alpha_{n}^{4 N+1}\right) z^{4 N+1}
$$

we obtain a polynomial in $U$ vanishing on $C$ precisely at the points $\alpha_{1}$, $\alpha_{2}, \ldots, \alpha_{n}$. Then

and

$$
P_{n}(z)=Q_{n}(z) \quad \text { if } \alpha_{n}^{4 N+1}=-1
$$

$$
P_{n}(z)=Q_{n}(z) \otimes\left(1+z-\alpha_{n}^{4 N+1} z^{2}\right) \quad \text { otherwise. }
$$

This completes the inductive and the proof of the lemma.

Lemma 2. If $n>3$, the polynomial $p_{n}(z)=1+z+z^{2}+\cdots+z^{n-1}-z^{n}+z^{n+1}+$ $\cdots+z^{2 n-1}$ does not vanish on $C$ and $\max \left\{\left|p_{n}(z)\right|: z \in C\right\}$ is attained if and only if $z=1$.

Proof. Since

$$
p_{n}(z)=\frac{z^{2 n}-1}{z-1}-2 z^{n}
$$

we see that

$$
p_{n}\left(e^{i 2 \theta}\right) e^{-i(2 n-1) \theta}=\frac{\sin 2 n \theta}{\sin \theta}-2 e^{i \theta}
$$

so that $p_{n}\left(e^{i 2 \theta}\right)=0$ implies that $e^{i \theta}$ is real, which implies in turn that $p_{n}\left(e^{i 2 \theta}\right)$ $=p_{n}(1)=2 n-2$, a contradiction.

Our statement about the maximum modulus property of $p_{n}(z)$ follows from the fact that $p_{n}^{2}(z)$ has positive coefficients.

Lemma 3. If $n>0$, the polynomial $q_{n}(z)=1+z+z^{2}+\cdots+z^{n}-z^{n+1}$ does not vanish on $C$.

Proof. One has $(1-z) q_{n}(z)=1-2 z^{n+1}+z^{n+2}$, which, by the triangle inequality, can vanish only if $1, z^{n+1}$ and $z^{n+2}$ are in the same direction; that is, here, $z=1$. But, obviously, $q_{n}(1) \neq 0$.

Lemma 4. If $P$ is any polynomial such that $P(\beta) \neq 0$, then there exists a polynomial $T$ in $U$ which does not vanish on $C$ and is such that $P \otimes T$ attains its maximum modulus on $C$ at $z=\beta$.

Proof. We can assume that $\beta=1$. Let $T=q_{m} \otimes r \otimes p_{n}$ where $q_{m}$ and $p_{n}$ are as in Lemmas 3 and 2 respectively, and $r(z)=1+e^{i \theta} z-z^{2}$ with $\theta \neq \pm \pi / 2 ; r(z)$ does not vanish on $C$ and $\operatorname{Im} r^{\prime}(1) / r(1)=2 \sin \theta$. Consider first $P^{*}=P \otimes q_{m} \otimes r$. If $M$ is the degree of $P$, then

$$
\frac{P^{* \prime}(1)}{P^{*}(1)}=\frac{P^{\prime}(1)}{P(1)}+(M+1) \frac{q_{m}^{\prime}(1)}{q_{m}(1)}+(M+1)(m+2) \frac{r^{\prime}(1)}{r(1)}
$$


and therefore

$$
\begin{aligned}
\operatorname{Im} \frac{P^{* \prime}(1)}{P^{*}(1)} & =\operatorname{Im} \frac{P^{\prime}(1)}{P(1)}+0+(M+1)(m+2) \operatorname{Im} \frac{r^{\prime}(1)}{r(1)} \\
& =\operatorname{Im} \frac{P^{\prime}(1)}{P(1)}+(M+1)(m+2) 2 \sin \theta .
\end{aligned}
$$

A proper choice of $m$ and $\theta$ will cancel this expression, making zero a stationary point of the function $\left|P^{*}\left(e^{i \theta}\right)\right|^{2}$. Since this function does not vanish at $\theta=0$, this stationary point can be transformed into the maximum point over all $\theta$ by multiplying $P^{*}$ by a delta-like function, in our case by encapsulating it with $p_{n}$ for suitably large $n$. Indeed, one has

$$
\left|p_{n}\left(e^{i \theta}\right)\right|^{2}=4-4 \frac{\sin n \theta}{\sin (\theta / 2)} \cos (\theta / 2)+\left(\frac{\sin n \theta}{\sin (\theta / 2)}\right)^{2} .
$$

By computing the second derivative, it is easily seen that $p_{n}(\theta)$ falls off like $e^{-c n \theta^{2}}$, and this shows that it is an adequate delta function for our purposes.

This completes the proof.

\section{REFERENCES}

1. J. S. Byrnes and D. J. Newman, Null steering employing polynomials with restricted coeffcients, IEEE Transactions on Antennas and Propagation 36 (1988), 301-303.

Prometheus Incorporated, 21 Arnold Avenue, Newport, Rhode Island 02840 